\title{
Temporal Notch activation through Notch1a and Notch3 is required for maintaining zebrafish rhombomere boundaries
}

\author{
Xuehui Qiu • Chiaw-Hwee Lim • Steven Hao-Kee Ho • \\ Kian-Hong Lee $\cdot$ Yun-Jin Jiang
}

Received: 8 April 2009 / Accepted: 10 July 2009 /Published online: 25 August 2009

(C) The Author(s) 2009. This article is published with open access at Springerlink.com

\begin{abstract}
In vertebrates, hindbrain is subdivided into seven segments termed rhombomeres and the interface between each rhombomere forms the boundary. Similar to the $\mathrm{D} / \mathrm{V}$ boundary formation in Drosophila, Notch activation has been shown to regulate the segregation of rhombomere boundary cells. Here we further explored the function of Notch signaling in the formation of rhombomere boundaries. By using bodipy ceramide cell-labeling technique, we found that the hindbrain boundary is formed initially in mib mutants but lost after 24 hours post-fertilization (hpf). This phenotype was more severe in $m_{i b}^{t a 52 b}$ allele than in $m i b^{t f i 91}$ allele. Similarly, injection of $s u(h)-\mathrm{MO}$ led to boundary defects in a dosage-dependent manner. Boundary cells were recovered in $\mathrm{mib}^{t a 52 b}$ mutants in the hdacl-
\end{abstract}

Communicated by M. Hammerschmidt

Electronic supplementary material The online version of this article (doi:10.1007/s00427-009-0296-6) contains supplementary material, which is available to authorized users.

X. Qiu • C.-H. Lim • S. H.-K. Ho • K.-H. Lee · Y.-J. Jiang $(\bowtie)$

Laboratory of Developmental Signalling and Patterning,

Genes and Development Division,

Institute of Molecular and Cell Biology,

A*STAR (Agency for Science, Technology and Research),

61 Biopolis Drive,

Singapore 138673, Singapore

e-mail: yjjiang@imcb.a-star.edu.sg

Y.-J. Jiang

Department of Biochemistry, National University of Singapore,

8 Medical Drive,

Singapore 117597, Singapore

Y.-J. Jiang

School of Biological Sciences,

Nanyang Technological University,

60 Nanyang Drive,

Singapore 637551, Singapore deficient background, where neurogenesis is inhibited. Furthermore, boundary cells lost sensitivity to reduced Notch activation from 15 somite stage onwards. We also showed that knockdown of notch 3 function in notch1a mutants leads to the loss of rhombomere boundary cells and causes neuronal hyperplasia, indicating that Notch1a and Notch3 play a redundant role in the maintenance of rhombomere boundary.

Keywords Notch · Zebrafish · Hindbrain .

Lateral inhibition $\cdot$ Neurogenesis

\section{Introduction}

During the zebrafish CNS development, the neural plate undergoes secondary neurulation and is converted into the neural keel, which subsequently develops into neural tube by cell detachment in the center (Schmitz et al. 1993; Kimmel et al. 1994). The anterior part of the neural tube further expands and swells mediolaterally, generating the forbrain, midbrain, and hindbrain. Later the hindbrain will be further subdivided into seven reiterated units, termed rhombomeres. Each rhombomere has its unique identity by expressing distinct gene profile, such as different combination of Hox genes. Rhombomeres have been shown to be involved in patterning neural crest cells and directing their migration to proper branchial arches (reviewed by Trainor and Krumlauf 2000). The interface between two rhombomeres is termed rhombomere boundary, which is formed by internal subdivision of the hindbrain according to a speciesspecific sequence. In zebrafish, the first rhombomere boundaries ( $\mathrm{r} 3 / 4$ and $\mathrm{r} 4 / 5)$ appear between five to seven somite stage (ss) and the last one appears at about 16 ss (Moens et al. 1998). Initially, segregation of rhombomeres 
results from different affinity between odd and even rhombomeres, which eventually acquire distinct identity (Guthrie and Lumsden 1991). Later, a finer scale cell sorting is accomplished by interactions between Eph receptor-expressing cells and Ephrin ligand-expressing cells (Xu et al. 1999). Recent studies have suggested that EphA4-dependent adhesion plays a redundant role with EphA4-dependent repulsion in rhombomere boundary formation (Cooke et al. 2005).

In Drosophila, it has been well studied that Notch signaling is involved in the dorsal-ventral boundary formation of wing disk (reviewed by Tepass et al. 2002). Fringe modulates the Notch sensitivity to different ligands and hence ensures that Notch activity is limited to the boundary cells. Recently, several studies have converged to provide evidence that Notch activation plays a similar role in the formation of rhombomere boundaries in a teleost, zebrafish. Firstly, large-scale genetic screen has identified several mutants that displayed rhombomere boundary defects, including mib mutants (Jiang et al. 1996). Positional cloning has revealed that mib encodes an E3 ligase required for efficient Notch activation in the neighboring cells by regulating ligand endocytosis (Itoh et al. 2003). Secondly, in deltaA dominant-negative mutants and $\mathrm{mib}$ mutants, strong neurogenic phenotype is observed and the rhombomere boundaries are disrupted (Riley et al. 2004; Appel et al. 1999; Cheng et al. 2004). Thirdly, further mosaic analysis has provided evidence that Notch activation is required for the regulation of specific rhombomere boundary cell movement: cells with high activation of Notch receptor or expressing a dominant-active form of $\mathrm{Su}(\mathrm{H})$ are prone to segregate into the boundary region, whereas cells expressing a dominant-negative form of $\mathrm{Su}(\mathrm{H})$ are expelled away from the boundary region (Cheng et al. 2004). In addition, $r f n g$ is expressed in the rhombomere boundaries and acts as a modulator involving in regulating neurogenesis (Qiu et al. 2004; Amoyel et al. 2005). Despite the mild neurogenic phenotype, no rhombomere boundary defect was observed in zebrafish notchla mutants, suggesting a redundancy of multiple Notch homologs in the zebrafish (Gray et al. 2001). Recent study in mice has demonstrated that persistent and high levels of Hes1 expression repress Mash1 expression in boundary regions and hence ensure the generation of neuron-free zones in boundaries (Baek et al. 2006). Loss of Hes1 together with Hes5 and Hes 3 resulted in ectopic neurogenesis and hence the disruption of rhombomere boundaries. On the other hand, Wnt signaling has been proved to interplay with Notch signaling in the rhombomere boundary formation (Riley et al. 2004; Amoyel et al. 2005). Forced expression of wnt1 partially rescues hindbrain patterning in mib mutants. Furthermore, similar to the regulatory network in Drosophila, Wnt1 regulates delta and proneural gene expression in non-boundary cells, and hence prevents them from acquiring boundary cell fate.

In this study we observed progressive changes in cell morphology and premature neuron differentiation in the hindbrain of mib mutants. Inhibition of neurogenesis in $m i b$ mutants leads to the recovery of rhombomere boundaries. We have also confirmed and further demonstrated that the disruption of rhombomere boundary is $\mathrm{Su}(\mathrm{H})$ - and dosagedependent. In addition, we established that Notch1a and Notch3 play a redundant role and their activation is required in boundary maintenance before $15 \mathrm{ss}$.

\section{Materials and methods}

Fish maintenance and mutant identification

Zebrafish maintenance and breeding were carried out as previously described (Kimmel et al. 1995). Embryos were raised at $28.5^{\circ} \mathrm{C}$ and the approximate stage is determined under the dissecting microscope.

hadc1 hil618 mutants (Golling et al. 2002) used for characterization were genotyped by using two pairs of primers, one pair derived from the lacZ gene ( $\mathrm{P} 1$ : 5'-ATCCTCTAGACTGCCATGG-3'; P2: 5'-ATC GTAACCGTGCATCTG-3') harbored by the viral vector for confirming insertion and the other from the genomic sequence flanking the exon 1 (P1: 5'-CCTACAGTGATGGAACCTG T-3'; P2: 5'-CGGTCCACAGTATGAAGCTA-3') for confirming the hdacl gene. Homozygous mib mutants were distinguished from siblings by their abnormal trunk morphology. Identification of $\mathrm{mib}^{\text {ta52b}}$; hadcl ${ }^{\text {hil618 }}$ double mutants in Fig. 3 was further sequenced by using intron 21 and intron 22 of the mib genomic sequence (P1: 5'-AGCTTGACAGGCGTAGCAACA-3'; P2: 5'-ACGAT TGAACGCTACGTCACACA-3'), where the T to G transversion leads to a change of Met (ATG) to Arg (AGG).

Heat shock induction

Embryos were given heat shock for $1 \mathrm{~h}$ at $39^{\circ} \mathrm{C}$ and later incubated at $28.5^{\circ} \mathrm{C}$ for further development. The embryos were fixed after they reached the desired stage and processed for in situ hybridization or immunohistochemistry. Using this heat shock method, no hindbrain boundary defects in any batch of the wild-type siblings were observed.

\section{Live embryo imaging}

Bodipy ceramide (Molecular Probes) was dissolved in dimethyl sulfoxide (DMSO) at a concentration of $5 \mathrm{mM}$ for stock solution. Dechorinated embryos were soaked into 
$200 \mu \mathrm{M}$ bodipy ceramide solution for $30 \mathrm{~min}$ in the dark. The embryos were then washed with egg water $3 \times 10 \mathrm{~min}$ each and mounted in wells in $1 \%$ low melting agarose for confocal microscopy. Confocal imaging was performed using a Zeiss LSM510 laser-scanning microscope (Cooper et al. 1999) and its images were analyzed using LSM software (Zeiss) and Photoshop 6.0 (Adobe).

Whole-mount in situ hybridization and immunohistochemical staining

In situ hybridization was performed as described (Qiu et al. 2004). For immunohistochemical staining, embryos between 12 and 48 hpf were fixed in either 4\% formaldehyde buffered with phosphate-buffered saline (PBS) or $2 \%$ trichloroacetic acid (TCA) (Sigma-Aldrich) for $4 \mathrm{~h}$ at room temperature (RT). After fixation, embryos were rinsed with PBS and then with distilled water, permeabilized with acetone treatment at $-20^{\circ} \mathrm{C}$ for 5 to $10 \mathrm{~min}$, rinsed again with distilled water and then PBS, and then blocked in PBDT (PBS with $0.1 \%$ Triton X-100 and 1\% DMSO) with $10 \%$ goat serum for $2 \mathrm{~h}$ at RT. After incubation in primary antibody overnight at $4^{\circ} \mathrm{C}$, embryos were then washed intensively and incubated in biotin-labeled secondary antibody for $2 \mathrm{~h}$ followed by soaking in a peroxidaseconjugated avidin-biotin complex (ABC kit; Pierce) for $1 \mathrm{~h}$. The following antibody and dilution were used: antiphospho-histone3 (Cell Signaling), 1:200 and anti-Hu (Molecular Probe), 1:1,000. A brown precipitate was formed by incubating embryos with $0.8 \mathrm{mg} / \mathrm{ml}$ of diaminobenzidine (DAB) (Sigma-Aldrich) and 0.001\% $\mathrm{H}_{2} \mathrm{O}_{2}$. For whole-mount immunofluorescent staining, after the primary antibody incubation and intensive wash, embryos were incubated in PBDT with goat anti-mouse IgG Alexa Fluo 488 (Molecular Probe) at 1:400 dilution at RT for $2 \mathrm{~h}$. Embryos were then rinsed with PBDT and analyzed using a Zeiss fluorescence microscope. Images were obtained using a Zeiss 510 confocal microscope.

Morpholino microinjections

Morpholinos were designed to target the translation or block the intron-exon splicing following the rule recommended by Gene Tools. The morpholinos were dissolved in $1 \times$ Danieu's buffer [ $58 \mathrm{mM} \mathrm{NaCl}, 0.7 \mathrm{mM} \mathrm{KCl,} 0.4 \mathrm{mM} \mathrm{MgSO} 4,0.6 \mathrm{mM}$ $\mathrm{Ca}\left(\mathrm{NO}_{3}\right)_{2}, 5.0 \mathrm{mM}$ HEPES ( $\mathrm{pH}$ 7.0)] to give a final stock concentration of $5.0 \mathrm{mM}$ as previously described (Nasevicius and Ekker 2000). For injection, the morpholinos were diluted in $1 \times$ Danieu's buffer at concentrations from 0.2 to $1.0 \mathrm{mM}$. One to two nanoliters of MO was injected into each embryo. Sequence for $s u(h)$-MO: 5'-CAAACTTCCCTGTCACA ACAGGCGC-3' (Sieger et al. 2003), sequence for notch $1 b$ exon 27 donor-MO: 5'-AATCTCAAACTGACC
TCAAACCGAC-3' (Milan et al. 2006), sequence for notch3-ATG-MO: 5'-ATATCCAAAGGCTGTAATTCC CCAT-3' (Lorent et al. 2004), sequence for notch3-3-MO: 5'-ATCAGTCATCTTACCTTCGCTGTTG-3', notch3-utrMO: 5'-ACATCCTTTAAGAAATGAATCGGCG-3' (Ma and Jiang 2007). Injected embryos of each individual notch3-MO displayed similar phenotypes and marker expression changes.

In vitro transcription and translation reactions

N-terminal notch3 cDNA was amplified by reverse transcription polymerase chain reaction (RT-PCR) using the forward primer 5'-ATCGATGGGGAATTACAGCCTTTGG-3' and the reverse primer 5'-ATCGATGCATGAGACACTCGT TGAC-3', based on the published notch3 cDNA sequence (NM_131549). After digested with ClaI, the PCR product was ligated to the pCS2+-myc vector. Six hundred nanograms of pCS2+-notch3-myc plasmid was added to TNT quick coupled transcription/translation reactions (Promega). Different amounts of notch3-ATG-MO were added into the reaction as indicated. Following incubation, the reaction products were run in a $10 \%$ acrylamide gel and detected by Western blot using antibody against Myc.

\section{Results}

Different mib mutants display a progressive defect in maintaining rhombomere boundaries with varying severity

Zebrafish $m i b$ gene encodes an E3 ligase that is an essential component of Notch signaling (Itoh et al. 2003). Previous studies have revealed that $m i b^{t a 52 b}$ mutants display rhombomere boundary defects by 26 hpf (Jiang et al. 1996) and that the neurogenic phenotype of $m i b^{t f i 91}$ is much weaker than that of mib $^{\text {ta52b }}$ (Zhang et al. 2007b). To address whether neurogenic defects affect the severity of boundary phenotype, we investigated the severity of rhombomere boundary defects in different mib alleles. In situ hybridization analysis of $r$ fng expression revealed that all rhombomere boundaries were affected in these two alleles (Fig. 1a-c). In wild-type embryos, rfng expression was restricted to the rhombomere boundaries (Fig. 1a), whereas in mib ${ }^{\text {ta52b }}$ mutants, the expression level of rfng was down-regulated and almost abolished in r4/5 and r5/6 boundaries (Fig. 1b). Different from $m i b^{t a 52 b}$ mutants, only lateral part of $r f n g$ expression was lost in mib ${ }^{t f i 91}$ mutants (Fig. 1c). As it has been demonstrated that rhombomeres are maintained as lineagerestricted compartments (Xu et al. 1999) and that distinctive cellular morphology can be observed in the successive rhombomere boundaries (Moens et al. 1996), we next examined the cellular changes in hindbrain region in 
different mib mutants. Bodipy ceramide labeling outlined cell contours and revealed the elongated epithelial structure of cells in the wild-type hindbrain (Fig. 1d, g). In mib ${ }^{\text {ta52b }}$ mutants, neuroepithelial cell organization was abnormal and cells were frequently rounder and also presented in aggregates separated by large intercellular spaces. These aggregates were often organized into rosettes that appear to cavitate and enclose a central lumen (Fig. 1e, h). In mib $b^{t f i 91}$ mutants, while the cellular shape appeared normal in the medial part of the hindbrain, cells became rounder in the lateral part and also had lost their epithelialized shape (Fig. 1f, i). These observations indicated that the disruption of rhombomere boundary in mib ${ }^{\text {ta52b }}$ mutants was more severe than that in $m i b^{t f i 91}$ mutants.

$\mathrm{N}$-cadherin ( $\mathrm{N}$-cad) is a cell adhesion molecule and was expressed at high level in the whole hindbrain in wild-type embryos at $15 \mathrm{ss}$ (Fig. 1s). In mib ${ }^{\text {ta52b }}$ mutants, the $n$-cad level was greatly down-regulated (Fig. 1t). In contrast, we did not detect obvious $n$-cad down-regulation in mib $^{t f i 91}$ mutants even at $22 \mathrm{hpf}$ (data not shown). Therefore, it implied that changes in the expression level of cell adhesion molecule could be one of the reasons accounting for the different degree of cellular changes between $m i b^{t a 52 b}$ and $m i b^{t f i 91}$ mutants. However, in $m i b^{\text {ta52b }}$ mutants at $15 \mathrm{ss}$, high level of $n$-cad expression in the boundaries flanking r5 was observed, suggesting that other adhesion molecules might be also required for the cell morphological change in mib mutants.

Recent studies have demonstrated that Notch activation is not sufficient for hindbrain boundary cell specification and loss of Mib function does not affect the initiation of rhombomere boundaries (Cheng et al. 2004). We provided further evidence to confirm that in $m b^{\text {ta52b }}$ embryos the boundaries were initiated despite the blockade of Notch activation. In $\mathrm{mib}^{\text {ta52b}}$ mutants at $17 \mathrm{hpf}$, elongated epithelial shape could be clearly identified and no obvious morphological difference between wild-type and mutant embryos could be detected in the hindbrain (Fig. 1k; compared to the control in j). Consistently, we still observed high level of foxb1.2 expression in the boundary cells of mib $^{\text {ta52b }}$ mutants at corresponding stage, despite its slight down-regulation in posterior boundaries (Fig. 1q; compared to the control in p). However, a few round cells were present and boundary cells could not be clearly distinguished from non-boundary cells in mib $^{\text {ta52b }}$ mutants by $22 \mathrm{hpf}$ (Fig. 1m; compared to the control in 1). Furthermore, the cellular change was most severe in $r 5$. We also found an increase of Hu-positive cells in the $\mathrm{r} 5$ region of $\mathrm{mib}^{\mathrm{ta52b}}$ mutants, suggesting that a loss of $\mathrm{r} 4 / \mathrm{r} 5$ and $\mathrm{r} 5 / \mathrm{r} 6$ boundaries is correlated with the strong neurogenic phenotype in the $\mathrm{r} 5$ region (Fig. 1n, o).

We have also checked whether cell proliferation and/or cell death attributes to the observed hindbrain phenotype in mib $^{\text {ta52b }}$ mutants. Fewer dividing cells were observed in $m_{i b} b^{t a 52 b}$ mutants when compared to their wild-type siblings. However, reduction of cell proliferation in wild-type embryos by aphidicolin treatment did not result in boundary disruption (Supplementary Fig. 1). Similarly, no obvious difference in apoptosis could be detected in wild-type and $m i b^{t a 52 b}$ embryos by using TUNEL staining (data not shown). These results indicated that decreased cell proliferation and apoptosis are unlikely responsible for the disruption of rhombomere boundaries in mib $^{\text {ta52b }}$ mutants.

Maintenance of rhombomere boundaries is $\mathrm{Su}(\mathrm{H})$ and dosage-dependent

The preceding analysis of boundary cell phenotype in $m i b^{t f i 91}$ and $m i b^{\text {ta52b }}$ mutants suggested that different level of compromise in Notch signaling has graded consequence in boundary formation and cell morphology. To address this issue more quantitatively, we injected different amount of $s u(h)-\mathrm{MO}$ into wild-type embryos and checked their hindbrain development. In addition to the loss of all somites posterior to the first five to seven somites (Sieger et al. 2003), irregular hindbrain morphology like mib ${ }^{\text {ta52b }}$ was observed (data not shown). Moreover, in $0.2 \mathrm{pmol} s u(h)$ MO-injected embryos $(n=12 / 13)$, rfng expression was reduced and most obviously at $\mathrm{r} 4 / \mathrm{r} 5$ and $\mathrm{r} 5 / \mathrm{r} 6$ boundaries (Fig. 2b; compared to the control in a). Using her4 as a Notch activation readout, we have observed that the expression level of her4 was greatly reduced in $s u(h)$ MO-injected embryos at $24 \mathrm{hpf}$ when compared to the wild-type control embryos $(n=22 / 22$; Fig. 2c, d). This analysis confirmed that Notch activation in rhombomere boundary maintenance is via $\mathrm{Su}(\mathrm{H})$ (Cheng et al. 2004).

We further injected $0.1 \mathrm{pmol}$ and $0.5 \mathrm{pmol} s u(h)-\mathrm{MO}$ into wild-type embryos and compared their effects on the disruption of rhombomere boundaries. Under both conditions, interfaces between rhombomeres could be easily identified at $17 \mathrm{hpf}$ (Fig. 2e, f; $n=4$ ), suggesting that the initiation of rhombomere boundaries were not affected in embryos injected with either dosage of $s u(h)$-MO. However, by 22 hpf obvious cellular shape change was observed in $\mathrm{r} 4$ and $\mathrm{r} 5$ region and the interstitial space increased in the lateral part of hindbrain (Fig. 2g, h). Furthermore, the change in 0.5 pmol $s u(h)$-MO-injected embryos was more severe than that in 0.1 pmol su(h)-MO-injected embryos. Next we fixed MO-injected embryos at different time points and processed for foxb1.2 in situ hybridization (Fig. 2i-k). While more than half the number of $0.1 \mathrm{pmol} s u(h)$-MOinjected embryos displayed wild-type like foxb1.2 expression pattern at $21 \mathrm{hpf}$, all the embryos injected with $0.5 \mathrm{pmol} s u(h)$-MO showed a change in foxb1.2 expression, though to a different degree. As we expected, by $26 \mathrm{hpf}$ the expression of foxb1.2 was down-regulated in embryos injected with either $0.1 \mathrm{pmol}$ or $0.5 \mathrm{pmol} s u(h)$-MO. 

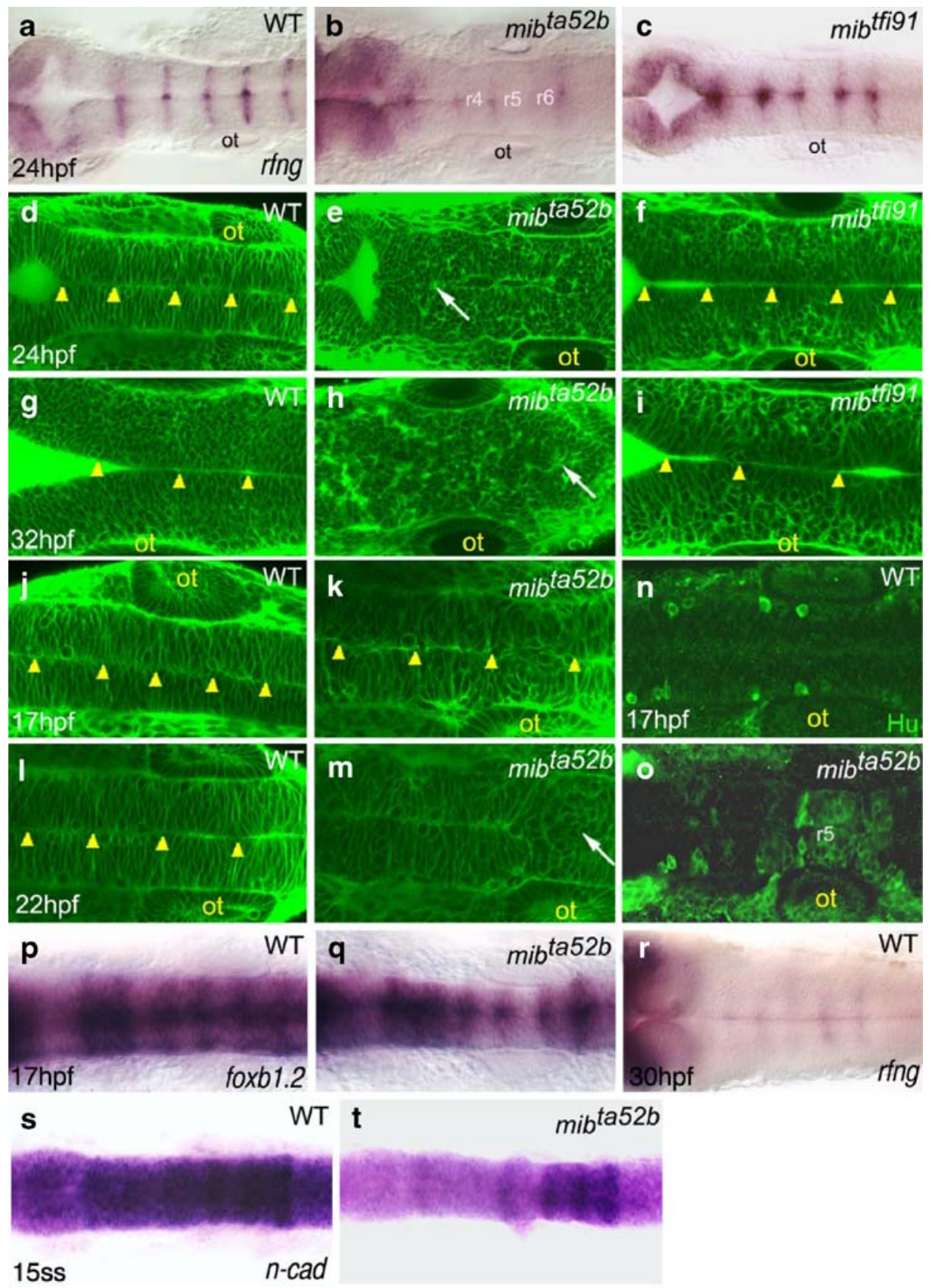

Fig. 1 Disruption of hindbrain rhombomere boundaries in mib mutants. a-c Representative $r f n g$ expression. a $r f n g$ is strongly expressed at rhombomere boundaries in wild-type embryos at $24 \mathrm{hpf}$. b In mib ${ }^{\text {tas2b }}$ mutants, almost no rfng transcripts can be detected at the $\mathrm{r} 4 / 5$ or $\mathrm{r} 5 / 6$ boundary and $r f n g$ is present at lower levels in the remaining boundaries. c In $m i b^{t f i 1}$ mutants, rffng expression remains intact in the medial part of the boundaries and disappears in the lateral part. $\mathbf{d}-\mathbf{m}$ Confocal microscopic images at hindbrain levels by using bodipy ceramide staining technique: wild-type siblings at d 24 hpf, g 32 hpf, j 17 hpf, and $\mathbf{I} 22 \mathrm{hpf}$; mib $^{\text {tas } 2 b}$ mutants at e $24 \mathrm{hpf}, \mathbf{h} 32 \mathrm{hpf}, \mathbf{k} 17 \mathrm{hpf}$, and m $22 \mathrm{hpf}$; and mib ${ }^{t f i 1}$ mutants at $\mathbf{f} 24 \mathrm{hpf}$ and $\mathbf{i} 32 \mathrm{hpf}$. White arrows indicate ectopic lumens in dorsal rosette-like structures in mib mutants. Rhombomere interfaces are marked with yellow arrowheads. Note that in mib mutants the cellular shapes are irregular, while in wild-type siblings cells are polarized and elongated. $\mathbf{k}$ The majority of cells in $m i b^{t a 52 b}$ mutants

display elongated epithelial structure at $17 \mathrm{hpf}$ and the interfaces between rhombomeres are visible. $\mathbf{m}$ Rosette-like structure can be found in $m_{i b} b^{t a 52 b}$ mutants at $22 \mathrm{hpf}$ and the boundary interface between $\mathrm{r} 4$ and $\mathrm{r} 5$ is hard to be identified at this stage. n, o Anti-Hu antibody, which recognizes the pan-neuronal marker $\mathrm{Hu}$, was used to identify $\mathrm{Hu}$-positive cells in $\mathbf{n}$ wild-type and $\mathbf{o} m i b^{t a 52 b}$ mutants at 17 hpf. Note that in $\mathrm{mib}^{\text {ta52b }}$ mutants the number of Hu-positive cells is greatly increased in the $\mathrm{r} 5$ region. $\mathbf{p}$ In wild-type embryos at $17 \mathrm{hpf}$, foxb1.2 is expressed at high level in the boundary cells. q Despite the slight down-regulation of foxb1.2 in the posterior hindbrain of mib ${ }^{\text {tas2b }}$ mutants at $17 \mathrm{hpf}$, high expression level of foxb1.2 still can be observed in the boundary cells. $\mathbf{r}$ Expression level of $r f n g$ is gradually reduced in embryos at $30 \mathrm{hpf}$. $\mathbf{s}$, $\mathbf{t}$ Expression of $n$-cad was greatly reduced in $\mathbf{t} m i b^{t a 52 b}$ mutants, when compared to $\mathbf{s}$ wild-type embryos at $15 \mathrm{ss}$. All images are dorsal views with anterior to the left. ot, otic vesicle 
Fig. 2 Lack of $\mathrm{Su}(\mathrm{H})$ by injecting $s u(h)-\mathrm{MO}$ causes a dosagedependent rhombomere boundary defect. Representative $r$ fng expression in a uninjected embryos or $\mathbf{b}$ embryos injected with $0.2 \mathrm{pmol}$ $s u(h)-\mathrm{MO}$ at $24 \mathrm{hpf}$. In $s u(h)$ morphants, $r f n g$ expression is significantly reduced, compared to wild-type uninjected embryos. c, d her 4 expression changes in $\mathbf{c}$ uninjected embryos and d 0.2 pmol su (h)-MO-injected morphants at 24 hpf. Note the reduction of her 4 expression level in injected embryos. e-h Confocal microscopic images at hindbrain levels by using bodipy ceramide staining technique. In embryos injected either with e 0.1 pmol or $\mathbf{f} 0.5 \mathrm{pmol}$ $s u(h)-\mathrm{MO}$ and observed at $17 \mathrm{hpf}$, the interfaces between rhombomeres can be easily identified and the cellular structure is elongated as wild-type. Yellow arrowheads indicate the interfaces between two adjacent rhombomeres. By $22 \mathrm{hpf}$, cells start to lose their epithelial structure in embryos injected with g $0.1 \mathrm{pmol} s u(h)$-MO. More severe effect is observed in the embryos injected with $\mathbf{h} 0.5 \mathrm{pmol} s u(h)$-MO. Note that in the $\mathrm{r} 4$ and $\mathrm{r} 5$ region, the cellular structure is irregular and the arrangement is disorganized. i $I-\mathbf{k} I I I$ Representative progressive disruption of the rhombomere boundaries in $s u(h)$-MO-injected embryos. Same batch of embryos injected with 0.1 pmol (affected embryo number shown in blue) or $0.5 \mathrm{pmol}$ (affected embryo number shown in red) and fixed at different time points, i $I-I I I 21 \mathrm{hpf}, \mathbf{j} I-I I I$ $24 \mathrm{hpf}$, and $\mathbf{k} I-I I I 26 \mathrm{hpf}$, for foxb1.2 in situ hybridization analysis in hindbrain. Variable foxb1.2 expression changes in $s u(h)$-MO-injected embryos can be classified into three classes: class I shows minor changes; class II displays less severe changes; class III displays the most severe changes. Elevated expression of foxb1.2 can be observed in the rhombomere boundaries of I wild-type embryos. foxb1.2 expression in $\mathbf{m} m i b^{t f i 91}$ and $\mathbf{n} m i b^{t a 52 b}$ mutants at $24 \mathrm{hpf}$. Note that foxb1.2 transcripts can be detected at the medial part of the boundary in $m i b^{t f i 91}$ mutants, while in $m i b^{t a 52 b}$ mutants the expression of foxb1.2 is uniformly at low level and no boundary expression is visible. her6 expression in o wild-type, $\mathbf{p} m i b^{t f i 91}$, and $\mathbf{q} m i b^{t a 52 b}$ embryos at $24 \mathrm{hpf}$. Note that her6 expression level is dramatically reduced in $m i b^{t a 52 b}$ as compared to that in $m i b^{t f i 91}$ mutants. her 9 expression in $\mathbf{r}$ wild-type, $\mathbf{s}$ $m i b^{t f i 91}$, and $\mathbf{t} m i b^{t a 52 b}$ embryos at 24 hpf. Note that her 9 expression level is decreased to a greater extent in $m i b^{\text {tas2b }}$ as compared to that in $m i b^{t i g 1}$ mutants. All panels are dorsal views with anterior to the left

Moreover, the severity of the foxb1.2 expression changes in these injected embryos at $26 \mathrm{hpf}$ was stronger than that of $m i b^{t f i 91}$ mutants and was comparable to that of $m i b^{\text {ta52b }}$ mutants (Fig. 2m, n). Thus, these observations suggested that the severity of the disruption in rhombomere boundaries of $s u(h)$-MO-injected embryos is dosage-dependent.

Early studies have shown that Notch activation is repressed in hindbrain to a greater extent in $\mathrm{mib}^{\text {ta52b }}$ mutants than in $m i b^{t f i 91}$ mutants by using her4 as a Notch activation readout (Zhang et al. 2007b). her6, mouse Hes 1 ortholog, is expressed in hindbrain and regulated by Notch signaling (Pasini et al. 2004). We used it to specifically test whether Notch is differentially compromised in hindbrain of different mib alleles. While her 6 was moderately reduced in $m i b^{t f i 91}$ mutants (Fig. 2o, p), it was greatly reduced in $m i b^{t a 52 b}$ mutants (Fig. 2q), demonstrating that Notch activation in hindbrain is decreased to a lesser degree in $m i b^{t f i 91}$ mutants when compared to $m i b^{t a 52 b}$ mutants, which is consistent with the data from the $s u(h)-\mathrm{MO}$ knockdown. her 9 , another Hes homologue, is expressed at high level in
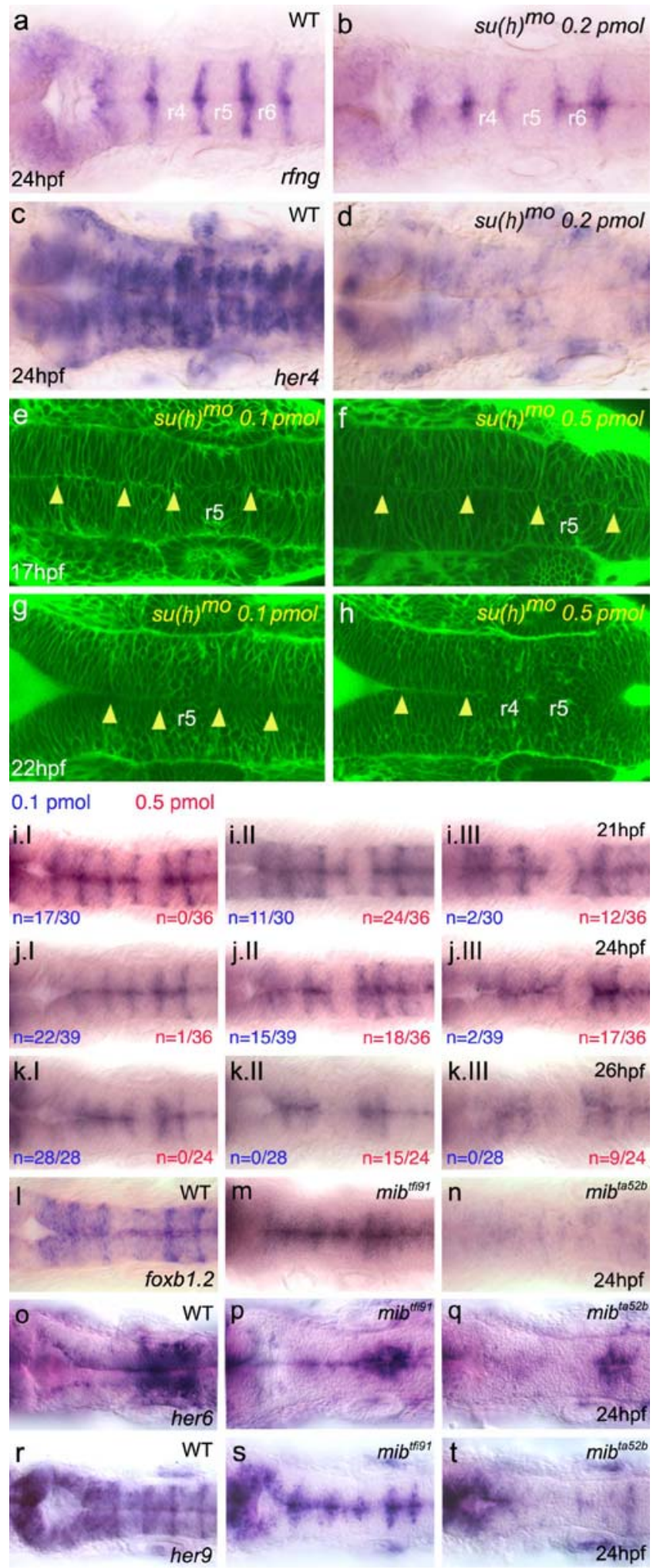

rhombomere boundaries and regulated by Notch signaling in pronephros ( $\mathrm{Ma}$ and Jiang 2007). Similarly, her9 expression was reduced to a greater extent in $m i b^{\text {ta52b }}$ mutants when compared to $m i b^{t f i 91}$ mutants (Fig. 2r-t). 
Partial rescue of rhombomere boundaries in hdach hil618. $^{\text {. }}$ $m i b^{\text {ta52b }}$ double mutants

Previous studies have shown that $h d a c l$ is required for the promotion of neurogenesis through repression of Notchactivated target genes (Cunliffe 2004). We next questioned whether the blockade of neurogenesis by hdacl deficiency in $m i b^{t a 52 b}$ mutants can restore the boundaries. Identification of hdacl ${ }^{\text {hil618 }}$ mutants, mib ${ }^{\text {ta52b }}$ mutants, and hdacl ${ }^{\text {hil618 }}$; $m i b^{\text {ta52b }}$ double mutants was confirmed both by PCR analysis of viral insertion and sequencing PCR products flanking the exon 21 of mib gene (Fig. 3a, b). At $24 \mathrm{hpf}$, expression level of $h u C$, a pan-neural marker, was dramatically increased in $m i b^{\text {tas2b }}$ mutants (Fig. 3c (2)). As $\mathrm{HuC}$ antibody staining has revealed that $\mathrm{r} 5$ is a region where neurons mature much earlier than other rhombomeres (Fig. 10), thus, it was not surprising to discover that the expression of neuronal markers, such as deltaA and $n g n l$, was enhanced in the hindbrain but not $\mathrm{r} 5$ region of mib ${ }^{\text {ta52b }}$ mutants (Fig. 3d (2), e (2)). Similar to rfng, expression of another boundary marker, foxb1.2, was greatly reduced in mib ${ }^{\text {tas2b }}$ mutants (Fig. 3f (2), g (2)). In hdacl ${ }^{\text {hil } 618}$ mutants, expression levels of $h u C$, delta $A$ and $n g n l$ were decreased to a great extent when compared to wild-type embryos (Fig. 3c (3), d (3), e (3)). However, the expression level of boundary markers, $r f n g$ and foxb1.2, was slightly reduced, though the pattern remained unperturbed, in hdacl $1^{\text {hil } 618}$ mutants (Fig. $3 \mathrm{f}$ (3), g (3)). In hdacl $1^{\text {hil618; }}$; mib ${ }^{\text {ta52b }}$ mutants, the expression level of $h u C$ was restored as compared to $\mathrm{mib}^{\text {ta52b }}$ mutants (Fig. 3c (1), c (2), c (4)). Similarly, the expression level and segmental pattern of deltaA and ngnl were also restored, though not completely, in double mutants when compared to mib $^{\text {ta52b }}$ mutants (Fig. 3d (1, 2, and 4), e (1, 2, and 4)). To determine whether the inhibition of neurogenesis in hdacl ${ }^{\text {hil618; }}$;ib ${ }^{\text {ta52b }}$ double mutants could rescue the boundary disruption in $m i b^{t a 52 b}$ mutants, expression of $r f n g$ and foxb1.2 was checked. The expression of $r f n g$ and foxb1.2 was increased and high expression level was observed in the middle region of rhombomere boundaries (Fig. $3 f(4)$, g (4)). These results suggested that upon inhibition of neurogenesis, boundary cells maintain their undifferentiated state.

Heat-shock-induced $d n-X S u(H)$ expression beginning before $8 \mathrm{~s}$ stage results in rhombomere boundary disruption

Rhombomere boundaries are transient structures during the hindbrain development. Previously, our results have shown that the expression of $r f n g$ is high at $24 \mathrm{hpf}$ (Qiu et al. 2004) and it will gradually disappear. We only observed low level of $r f n g$ expression by $30 \mathrm{hpf}$ (Fig. 1r) and no rfng expression was detected by $36 \mathrm{hpf}$. Next, to determine the time period required for Notch activation in rhombomere boundary maintenance, we used $\operatorname{Tg}(h \operatorname{sp} 70: X \operatorname{dnSu}(H)$ $m y c)^{\text {vu21 }}$ transgenic line (Latimer et al. 2005) to conditionally block Notch signaling. Heat shock beginning at tail bud (tb) to15 ss led to transgenic embryos with neurogenic phenotypes similar to the Notch deficient embryos by checking $h u C$ expression level $(n=16 / 16,10 / 10$, and 9/9 for tb, 8 ss and 15 ss, respectively; Fig. 4e, h, k; compared to the control in b). However, upon further investigation, we found that embryos heat-shocked at tb $(n=8 / 8)$ and 8 ss $(n=6 / 6)$ show a more significant reduction in her 4 expression than those heat-shocked at 15 ss $(n=9 / 9$; Fig. 4f, i, 1; compared to the control in c). These results suggested that Notch activation was blocked to a greater extent in embryos heat-shocked at tb and $8 \mathrm{ss}$. Consistently, the $r f n g$ expression was attenuated significantly in embryos heat-shocked at both stages, indicating a blockade of Notch activation starting from tb $(n=10 / 10)$ to $8 \mathrm{ss}(n=11 / 11)$ was adequate to disrupt the boundaries by $24 \mathrm{hpf}$ (Fig. $4 \mathrm{~d}, \mathrm{~g}$ ). Embryos heat-shocked after 15 ss $(n=23 / 23)$ only showed neurogenic phenotype but the boundary marker was robustly expressed as wild-type embryos at $24 \mathrm{hpf}$ (Fig. 4j). We thus conclude that hindbrain boundary cells lose sensitivity to a blockade of Notch activation between 8 ss to 15 ss.

Knockdown of Notch3 function in notchla mutants results in a loss of rhombomere boundary cells and neuronal hyperplasia

Previous studies have shown that notchla, notchlb, and notch 3 are expressed in the hindbrain (Westin and Lardelli 1997). Therefore, it is of interest to identify the receptors involved in the maintenance of rhombomere boundary cell fate. To determine this, we injected published notch $1 b$ or notch 3 morpholino into notch $1 a^{\text {th } 35 b}$ mutants. The specificity of notch3-ATG-MO has been confirmed as previously published (Fig. 5a; Lorent et al. 2004). Interestingly, notch $1 b$ and notch 3 morphants, similar to notchla (deadly seven) mutants, did not show a rhombomere boundary phenotype. Similar to mib $b^{\text {tas2b }}$ mutants, we found that cells aggregate and organize into rosette-like structures in the posterior hindbrain in notch3-MO-injected notch $1 a^{\text {th } 35 b}$ mutants (Fig. 5b, c; $n=4$ ). Furthermore, in notch3-MOinjected notch $1 a^{\text {th } 35 b}$ mutants, we observed a great reduction of boundary cells by using rfng or foxb1.2 as rhombomere boundary markers (Fig. $5 \mathrm{~d}-\mathrm{g} ; n=18 / 20$ for e, $n=22 / 23$ for g). By contrast, we did not observe a loss of rhombomere boundary cells in the notchlb-MO-injected notch $1 a^{\text {th } 35 b}$ mutants (data not shown). Consistent with the loss of Notch activation, we observed that her 4 was obviously down-regulated in notch3-MO-injected notch1$a^{\text {th } 35 b}$ mutants at $15 \mathrm{hpf}(n=12 / 12$, Fig. $5 \mathrm{j}, \mathrm{k})$. In addition to the boundary disruption, we also noted that the neural keel was irregular and the hindbrain appeared highly disorga- 

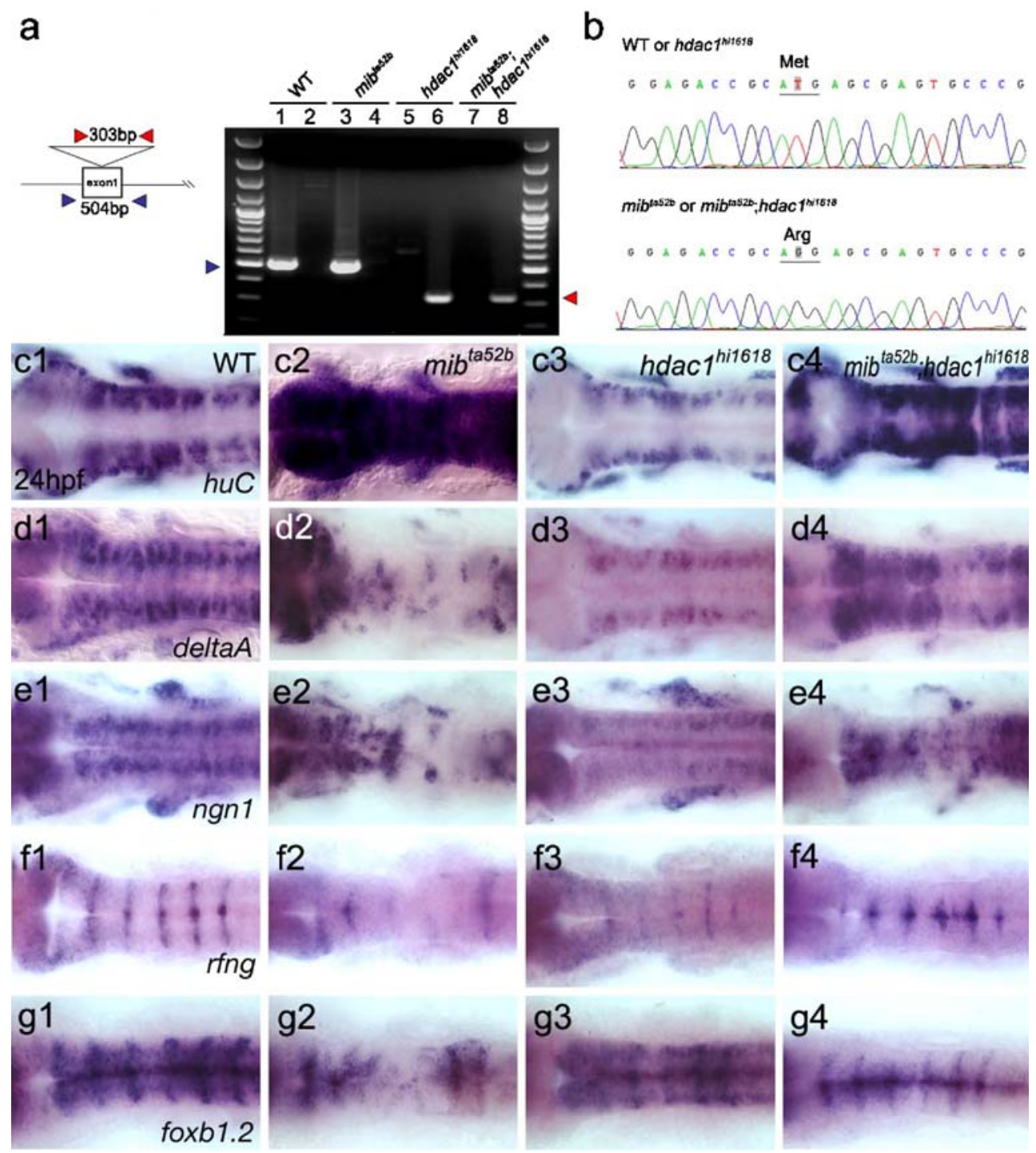

Fig. 3 Loss of Hdac1 function in the mib ${ }^{\text {tas2b }}$ mutants results in partial rescue of the rhombomere boundary defects. a Genotyping $h_{d a c l}{ }^{\text {hil618 }}$, $m i b^{\text {ta52b }}$, and $h d a c l^{\text {hil618 }}$;mib ${ }^{\text {ta52b }}$ embryos. Schematic drawing shows the insertion site of the viral vector in hdacl gene. The positions of the two pairs of primers used for genotyping the mutant embryos are indicated and their PCR products are shown in the gel image. Lanes 1, 3, 5, and 7 use primer set indicated in blue arrowheads while lanes 2, 4, 6, and 8 use primer set indicated in red arrowheads. Red arrowheads represent the primer pair for checking the viral insertion in homozygous $h_{\text {dacl }}^{\text {hil618 }}$ mutants and hdacl ${ }^{\text {hil618 }}$; mib ${ }^{\text {tas2b }}$ double mutants; blue arrowheads for checking the wild-type hdacl gene in the wild-type and mib ${ }^{\text {ta52b }}$ embryos. b In mib ${ }^{t a 52 b}$ mutants and hdac1 ${ }^{\text {hil618 }}$; mib $b^{\text {tas2b }}$ double mutants,

sequence analysis reveals that a $\mathrm{T}$ to $\mathrm{G}$ transversion caused an amino acid change from methionine to arginine. c-g Representative expression changes of marker in the mib ${ }^{\text {tas2b }}$, hdacl ${ }^{\text {hilo18 }}$, and mib $^{\text {tas2b }}$; hdacl hil618 mutants. In $h d a c 1^{\text {hil618 }}$ mutants, reduction in the expression level of $\mathbf{c}$ $h u C, \mathbf{d}$ deltaA, and e $n g n l$ indicated the inhibition of the neurogenesis when compared to wild-type embryos. Note that in $m i b^{\text {ta52b }}$ mutants, neuronal hyperplasia resulted in the severe disruption of rhombomere boundaries as indicated by the expression of boundary markers $\mathbf{f} r f i n g$ or $\mathbf{g}$ foxb1.2. Moreover, the boundary disruption in double mutants was milder than that of the mib ${ }^{\text {ta52b }}$ mutants. Note that all embryos were at $24 \mathrm{hpf}$ stage and anterior to the left

nized in notch3-MO-injected notch $1 a^{\text {th } 35 b}$ mutants (data not shown). These studies implied that Notch activation through two redundant receptors, Notch1a and Notch3, is required for the fate maintenance of rhombomere boundary cells by regulating their differentiation timing. Thus we further analyzed the neuron formation in notch3-MOinjected notchl $a^{\text {th } 35 b}$ mutants and found that cells in the

hindbrain are differentiated into neurons as $m i b^{t a 52 b}$ mutants (Fig. 5i; $n=18 / 18$ ). The dramatic increase in $h u C$ positive cells further confirmed the neurogenic phenotype in the notch3-MO-injected notch $1 a^{\text {th } 35 b}$ mutants (Fig. $5 \mathrm{~m}$; $n=31 / 31$ ). These results suggested that Notch1a and Notch3 play a redundant role in the late differentiation of rhombomere boundary cells. 


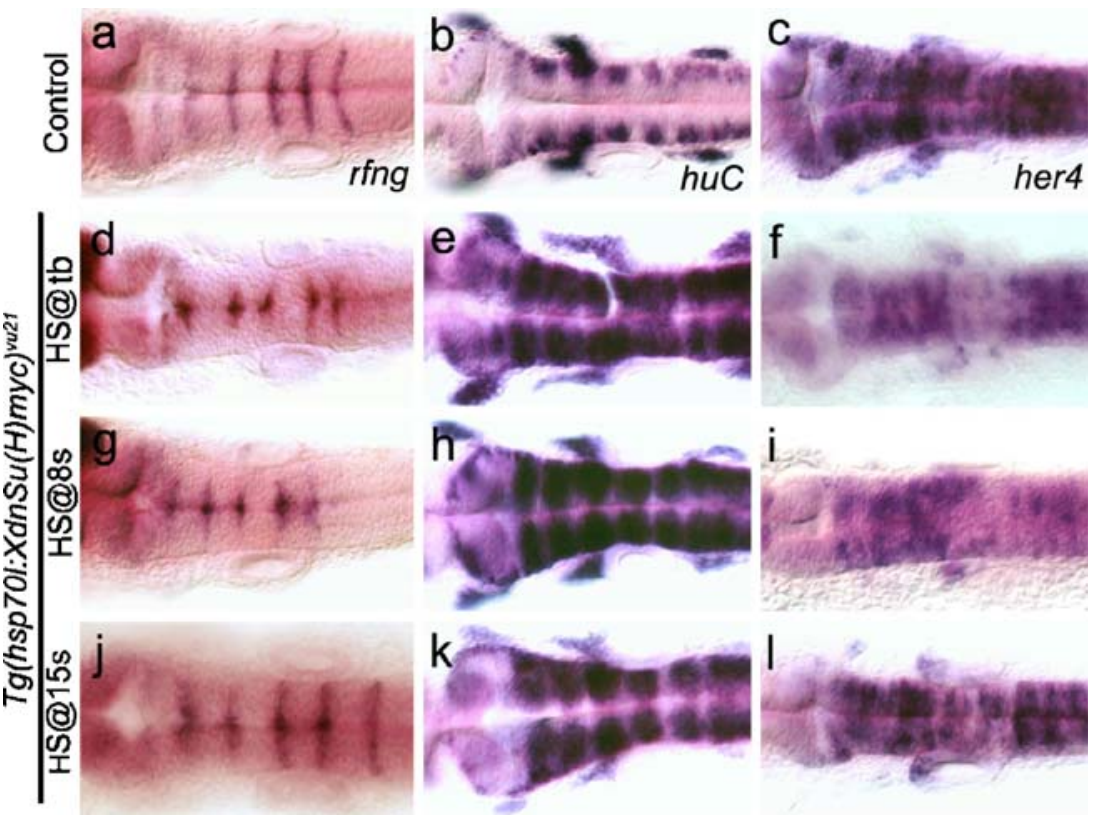

Fig. 4 Temporal requirement of Notch signaling for the maintenance of rhombomere boundaries at segmentation stage. a-c Wild-type and d-I $\operatorname{Tg}(h s p 70: X d n S u(H) m y c)^{v u 21}$ embryos heat-shocked at $\mathbf{d}-\mathbf{f}$ tail bud stage, $\mathbf{g}-\mathbf{i} 8$ ss, and $\mathbf{j}-\mathbf{I} 15$ ss. Note that in transgenic embryos heatshocked at $\mathbf{d}$ tail bud stage and $\mathbf{g} 8$ ss, the expression of boundary marker $r f n g$ is decreased considerably compared to a wild-type heatshocked embryos, whereas the expression level of $r f n g$ is almost unaffected in the transgenic embryos heat-shocked at $\mathbf{j} 15$ ss. In all $\mathrm{Tg}$ (hsp 70:XdnSu(H)myc) ${ }^{v u 21}$ heat-shocked embryos; e, h, k huC expres- sion level is greatly increased, whereas her4 expression is downregulated to various extent in $\mathbf{f}, \mathbf{i}, \mathbf{l}$, indicating that the neuron overpopulation in heat-shocked embryos results from the loss of Notch activation. Note that her4 expression in the posterior part of hindbrain is diminished to a greater extent compared to that in the anterior part of all the $\mathbf{f}, \mathbf{i}, \mathbf{I}$ heat-shocked $\operatorname{Tg}(h s p 70: X \operatorname{dnSu}(H) m y c)^{v u 21}$ embryos. All embryos are at $24 \mathrm{hpf}$ and all panels are dorsal views with anterior to the left

\section{Discussion}

$\mathrm{Su}(\mathrm{H})$-dependent Notch activation is required for the maintenance but not initiation of rhombomere boundaries by suppressing premature neuronal differentiation

Rhombomere boundary cells can be easily identified at $17 \mathrm{hpf}$ in $s u(h)$-MO-injected embryos or mib mutants. Therefore it is unlikely that $\mathrm{Su}(\mathrm{H})$-dependent Notch activation is required for the initiation of hindbrain boundary. Consistent with this, weak $r f n g$ expression was detected in the rhombomere boundaries in $m i b^{t a 52 b}$ mutants at $16.5 \mathrm{hpf}$ (Cheng et al. 2004). It has been shown that some residual Notch activation could be observed in mib mutants or $s u(h)$ morphants by using her4 as a Notchactivated target gene (Itoh et al. 2003; Zhang et al. 2007b). This observation suggests that the residual Notch activation might be through a $\mathrm{Su}(\mathrm{H})$-independent signaling that requires a cytoplasmic RING finger protein, Deltex (Le Borgne et al. 2005). However, knockdown of a hindbrainexpressing Deltex, deltex1 (DQ497599), did not lead to detectable hindbrain defects. Though the $s u(h)$-MO we used targeting two known $s u(h)$ genes (Echeverri and Oates 2007; Zhang et al. 2007b), it still remains possible that maintenance of rhombomere boundaries is entirely through the $\mathrm{Su}(\mathrm{H})$-dependent Notch activation, as morpholino injection may be unable to completely knock down $s u(h)$.

How does the boundary get lost? One possibility is that cell intermixing between rhombomeres is mis-regulated and hence the boundary cells cannot be distinguished. However, the expression of krox-20 is still very prominent at $20 \mathrm{hpf}$ and is only greatly reduced by $24 \mathrm{hpf}$ (Bingham et al. 2003). Yet we observed that the boundary cells become indiscernible from $17 \mathrm{hpf}$ in $m i b^{t a 52 b}$ mutants. In $m i b^{\text {tfigl }}$ mutants, krox-20 expression is normal at $24 \mathrm{hpf}$ and therefore cell intermixing is less likely accounted for the disruption of lateral rhombomere boundaries. We also observed reduced cell proliferation in the hindbrain region from $20 \mathrm{ss}$ in mib $^{\text {tas2b }}$ mutants. However, when we blocked the cell proliferation in wild-type embryos we found that the expression of boundary marker was normal. Therefore, reduced cell proliferation is unlikely to be a cause of disruption of hindbrain boundaries.

An alternative possibility is the premature differentiation of boundary cells into neurons. Detection of a pan-neuronal marker $h u C$ revealed that ectopic neurons in $24 \mathrm{hpf}$ embryos fills almost the whole hindbrain (Fig. 3c (2); Cheng et al. 2004). Also, we found that Hu-positive cells 
Fig. 5 Loss of Notch3 function in notch $1 a^{\text {th } 35 b}$ mutants results in rhombomere boundary disruption and neuronal hyperplasia. a The notch3 ATG-MO can specifically block translation of notch 3 expression constructs (containing ATG site) in vitro by TNT reactions in a dose-dependent manner. b, c Confocal microscopic images at hindbrain levels by using bodipy ceramide staining technique at $24 \mathrm{hpf}$. Note that interfaces between rhombomeres (yellow arrowheads) can be clearly identified in uninjected embryos, whereas the interface between $\mathrm{r} 4$ and $\mathrm{r} 5$ becomes obscure in notch3-MO-injected notch $1 a^{\text {th } 35 b}$ mutants. Also the irregular cellular shape changes can be easily found in $\mathrm{r} 5$ region (white arrow). $\mathbf{d}-\mathbf{i}$ In situ hybridization for boundary marker $\mathbf{d}$, e $r f n g$ and $\mathbf{f}, \mathbf{g}$ foxb1.2, and for proneural marker $\mathbf{h}, \mathbf{i}$ ngnl at $24 \mathrm{hpf}$. Note that the expression level of $r f n g$ or foxb1.2 is greatly reduced while the expression level of $n g n 1$ is greatly increased, indicating that the loss of boundary cells in notch3-MOinjected notch $1 a^{\text {th } 35 b}$ mutants results from early differentiation of boundary cells. In situ hybridization for a Notch activation readout marker $\mathbf{j}$, $\mathbf{k}$ her4 and postmitotic neuronal marker $\mathbf{l}, \mathbf{m} h u C$. Note that her4 was down-regulated in notch3-MO-injected notch $1 a^{\text {th } 35 b}$ mutants after loss of Notch activation at $15 \mathrm{hpf}$. Also note that more $\mathrm{huC}$ positive cells were present, indicating the neurogenic phenotype in notch3-MO-injected notch $1 a^{\text {th } 35 b}$ mutants at $24 \mathrm{hpf}$. All panels are dorsal views with anterior to the left

were increased to a great extent in $\mathrm{r} 5$ region at $17 \mathrm{hpf}$ in mib $^{\text {ta52b }}$ mutants. This correlates well with the earliest loss of $\mathrm{r} 4 / 5$ and $\mathrm{r} 5 / \mathrm{r} 6$ boundaries. It has been shown that CBF1, a mammalian $\mathrm{Su}(\mathrm{H})$ ortholog, binds to the HDAC1containing SMRT corepressor complex before its Notchdependent switch from a transcriptional repressor to an activator (Kao et al. 1998). Studies in zebrafish further revealed that hadcl is required for repressing Notch activation and the abundance of $a s h 1 b$ and ngn 1 transcripts is dramatically reduced at $26 \mathrm{hpf}$ in the hindbrain of hdacl morphants (Cunliffe 2004). Indeed, we observed that boundary cells were partially rescued in hdact hil618; mib $^{\text {ta52b }}$ double mutants.

There are several interpretations for the different severity between $m i b^{t a 52 b}$ and $m i b^{t f i 91}$ mutants. Recent reports have shown that Mib can interact with Mib2 and injecting mib2MO can further enhance the $m i b^{t f i 91}$ phenotype (Zhang et al. 2007a). Additionally, we observed that wnt1 expression was disorganized in $m_{i b}^{t a 52 b}$ mutants while in $m i b^{t f i 91}$ mutants the wntl expression pattern was still similar to that of wild-type embryos (data not shown). Thus we speculated that Wnt signaling was affected to a great extent in $m_{i b} b^{t a 52 b}$ mutants. Loss/reduction of Notch activation together with the reduction of Wnt signaling results in the severe disruption in rhombomere boundaries in $m_{i b}^{t a 52 b}$ mutants. Consistent with this interpretation, heat shock-driven wnt1 expression in mib $^{\text {ta52b }}$ mutants partially rescued the hindbrain boundary phenotype (Riley et al. 2004). Moreover, we observed that $n$-cad was significantly reduced in mib $^{\text {ta52b }}$ mutants and this might partially explain why cellular shape changes in $\operatorname{mib}^{t a 52 b}$ were more severe when compared to the mib $b^{t f i 91}$ mutants. However, it remains

\section{a}

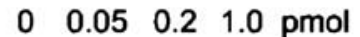
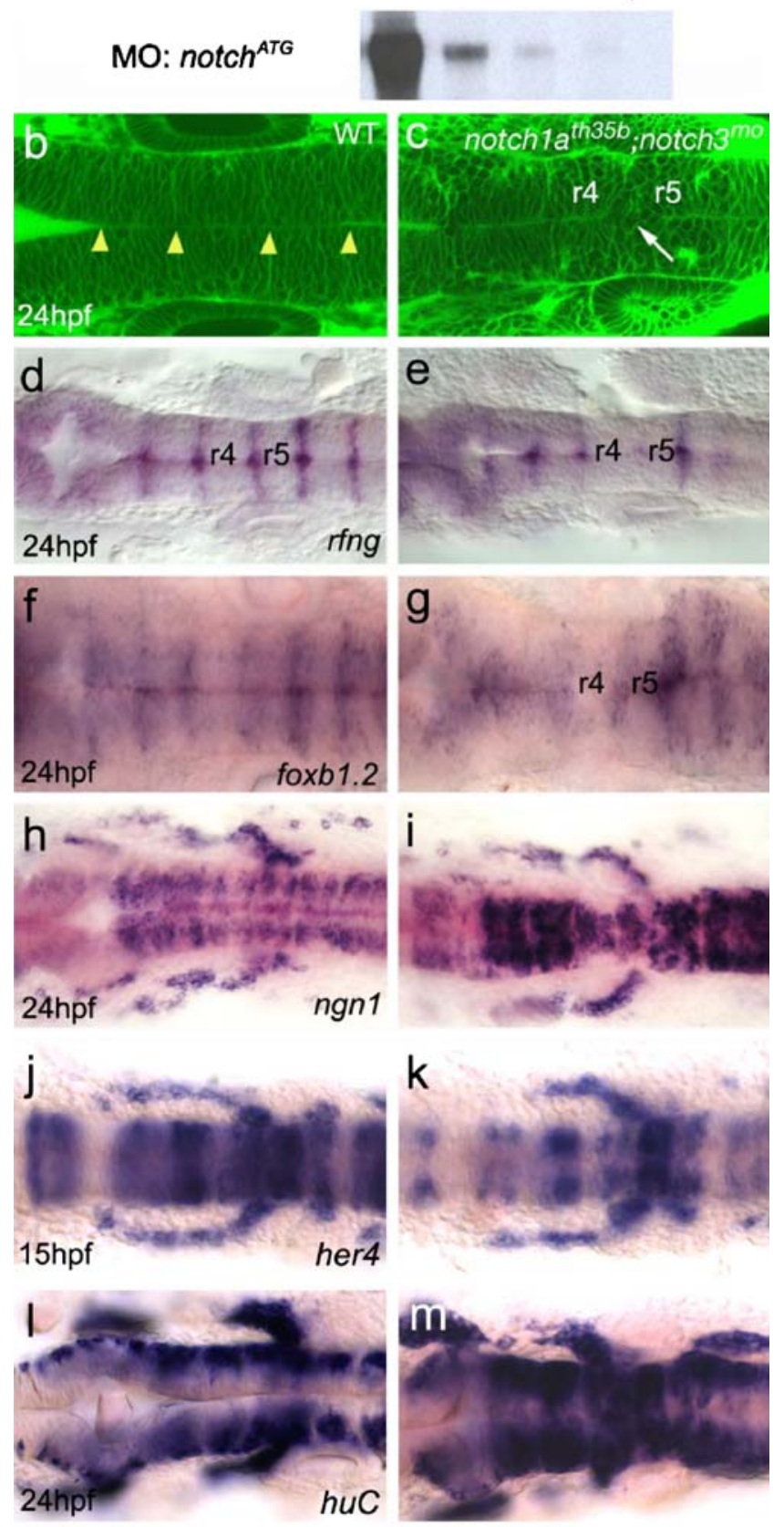

unanswered why the medial part of rhombomere boundary is more robust than in the lateral part.

Hindbrain boundary formation displays a low sensitivity to the level of Notch activation

notchla/des, deltaD/aei, and deltaC/bea mutants show somite defect and mild neurogenic phenotype (Gray et al. 2001; Holley et al. 2000; Jülich et al. 2005), whereas mib mutants exhibit somite abnormality, strong neurogenic phenotype, and disruption of rhombomere boundaries. Previous reports have indicated that her 4 can be used as a 
readout of Notch activation and we found that her 4 expression is normal in notchla mutants, while its expression is greatly reduced in $m^{t a 52 b}$ mutants (Takke et al. 1999; Zhang et al. 2007b). In addition, apart from her4 expression level, expression level of her6 and her 9 was also higher in $m i b^{t f i 91}$ mutants than that in $m i b^{t a 52 b}$ mutants. Consistent with this, the boundary disruption in $m i b^{t f i 91}$ is milder than that in $m i b^{t a 52 b}$ mutants. Furthermore, the higher the $s u(h)$-MO dosage was injected, the more severe the boundary defect was observed. These results suggest that hindbrain boundary has a lower functional threshold requirement for Notch activation than somite segmentation and neurogenesis, since only the embryos with severely compromised Notch activation show all the phenotypes, including rhombomere defects. Previously, it has been shown in mice that a low threshold for Notch activity is required to maintain proper somite segmentation (Huppert et al. 2005). Taken together, it seems that hindbrain boundary formation has the lowest threshold among all the known Notch-dependent processes.

While $\operatorname{Tg}(h s p 70: X d n S u(H) m y c)^{v u 21}$ embryos heatshocked after 15 ss maintained rfng expression at $24 \mathrm{hpf}$, embryos heat-shocked at 8 ss impaired its expression at hindbrain boundaries. Therefore, we conclude that boundary cells lose sensitivity to reduced Notch activation between 8 to 15 ss. Previous studies have suggested that boundary markers are up-regulated at $16-17 \mathrm{hpf}$, which is later than the appearance of morphological boundaries. It is also observed that the neurogenic phenotype of $\operatorname{Tg}(\mathrm{hsp} 70$ : $X d n S u(H) m y c)^{v u 21}$ embryos heat-shocked after 15 ss is comparable to that of notch3-MO-injected notch $1 a^{\text {th } 35 b}$ mutants. Therefore, it is unlikely that the presence of boundaries in these embryos heat-shocked after 15 ss is due to insufficient reduction in Notch signaling. Thus, our results suggested that the rhombomere boundaries became stable only after the Notch controlling timing of cell differentiation in boundary cells is properly executed.

Notch1a and Notch3 function redundantly in maintaining rhombomere boundary cell fates

notch $1 a$, notch $1 b$, and notch 3 were expressed in the entire hindbrain and the expression level was reduced in $m i b^{\text {ta } 52 b}$ mutant embryos. Previous studies have shown that both Notch1 and Notch3 have the same effects on astroglial development and HES-1 expression (Tanigaki et al. 2001). Overexpression of activated murine Notch 1 and Notch3 in transgenic mice blocks mammary gland development and induces mammary tumors ( $\mathrm{Hu}$ et al. 2006). However, the defects of Notch1 signaling accelerate the differentiation of pancreatic endocrine cells, while overexpression of the intracellular region of Notch3 induces the same phenotypes (Apelqvist et al. 1999). Biochemical analysis has further revealed that Notch3IC can compete with Notch1IC for binding to the RBP-JK and thus acts as a repressor of Notch1IC-mediated HES activation (Beatus et al. 1999). These observations seem controversial but can be reconciled by the explanation that different tissues/organs have their specific multi-potent progenitors or have their specific repertoire of coactivators or co-repressors. In zebrafish hindbrain, our observation that notch3-MO-injected notch $1 a^{\text {th } 35 b}$ mutants displayed strong neurogenic phenotypes (Fig. 5i, m), suggesting that notchla and notch3 play a redundant role in the neuron differentiation. Therefore, these results indicate that both Notch1a and Notch3 activation are required for the maintenance of boundary cells in undifferentiating state at segmentation stage. Our recent studies on zebrafish kidney and ionocyte formation demonstrated that Notchla and Notch3 play a redundant role in these organs as well (Ma and Jiang 2007; Hsiao et al. 2007), suggesting that a similar mechanism is utilized in the hindbrain. However, it remains unclear what is the exact function of Notch1b. One possible scenario is that its activation is involved in other developmental events rather than neurogenesis.

deltaA, deltaB, deltaD, and jaggedla are segmentally expressed in hindbrain (Haddon et al. 1998; Appel and Eisen 1998; Dornseifer et al. 1997; Zecchin et al. 2005). However, deltaA insertional mutants (hi781 and hi840), deltaD mutants (tr233), and their double mutants have normal rfng expression, which suggests that deltaA and deltaD are not involved in boundary cell differentiation or that the failure of exhibiting a phenotype is due to the redundancy of deltaB and/or jaggedla.

It has been shown that mouse Hes1 is expressed at high level in boundary regions and at low level in non-boundary regions (Baek et al. 2006). Similarly, we identified a Hairy and Enhancer of split homolog in zebrafish, her9, and found that it also shows persistent high expression level in the hindbrain boundary. However, knockdown of her 9 alone did not result in the loss of boundary cells. Although Hes1-null mice did not exhibit defects in nervous system, the inter-rhombomere boundaries are initiated normally at E9.5 but become ambiguous at E10.5 in Hes1;Hes5 doublenull embryos. In Hes1;Hes3;Hes5 triple-null mice the boundary defects become more significant, indicating that Hes1, Hes3, and Hes5 are all involved in regulating the boundary formation (Hatakeyama et al. 2004). In agreement with this, her3 (mouse Hes3 ortholog), her4, her6 (mouse Hes1 ortholog), her8a, her9, and her12 (mouse Hes5 homolog) have been identified to be expressed in the hindbrain (Hans et al. 2004; Takke et al. 1999; Pasini et al. 2001; Leve et al. 2001; Gajewski et al. 2006). Therefore, we speculate that similar to mice some her genes alone or in combination may play a role in maintaining boundary cells in an undifferentiated state. This warrants future study. 
Acknowledgements We are grateful to Wanyu Li, Tong Liang Tan, Ker Wai Wong, and Doreen Xin Yi Tey for their technical assistance; to Derryn Chan for the help in revising the manuscript and to members of the Jiang lab for stimulating and constructive discussions. We also thank Drs. Bruce Appel and Nancy Hopkins for supplying $T g(h s p 70: X d n S u(H)$ myc) ${ }^{\text {vu21 }}$ transgenic line and hadcl ${ }^{\text {hilo18 }}$ mutants, respectively. This work was supported by the Biomedical Research Council of A*STAR (Agency for Science, Technology and Research), Singapore.

Open Access This article is distributed under the terms of the Creative Commons Attribution Noncommercial License which permits any noncommercial use, distribution, and reproduction in any medium, provided the original author(s) and source are credited.

\section{References}

Amoyel M, Cheng YC, Jiang Y-J, Wilkinson DG (2005) Wnt1 regulates neurogenesis and mediates lateral inhibition of boundary cell specification in the zebrafish hindbrain. Development 132:775-785

Apelqvist Å, Li H, Sommer L, Beatus P, Anderson DJ, Honjo T, Hrabe de Angelis M, Lendahl U, Edlund H (1999) Notch signalling controls pancreatic cell differentiation. Nature 400:877-881

Appel B, Eisen JS (1998) Regulation of neuronal specification in the zebrafish spinal cord by Delta function. Development 125:371-380

Appel B, Fritz A, Westerfield M, Grunwald DJ, Eisen JS, Riley BB (1999) Delta-mediated specification of midline cell fates in zebrafish embryos. Curr Biol 9:247-256

Baek JH, Hatakeyama J, Sakamoto S, Ohtsuka T, Kageyama R (2006) Persistent and high levels of Hes1 expression regulate boundary formation in the developing central nervous system. Development 133:2467-2476

Beatus P, Lundkvist J, Oberg C, Lendahl U (1999) The Notch 3 intracellular domain represses Notch 1-mediated activation through Hairy/Enhancer of split (HES) promoters. Development 126:3925-3935

Bingham S, Chaudhari S, Vanderlaan $\mathrm{G}$, Itoh $\mathrm{M}$, Chitnis $\mathrm{A}$, Chandrasekhar A (2003) Neurogenic phenotype of mind bomb mutants leads to severe patterning defects in the zebrafish hindbrain. Dev Dyn 228:451-463

Cheng Y-C, Amoyel M, Qiu X, Jiang Y-J, Xu Q, Wilkinson DG (2004) Notch activation regulates the segregation and differentiation of rhombomere boundary cells in the zebrafish hindbrain. Dev Cell 6:539-550

Cooke JE, Kemp HA, Moens CB (2005) EphA4 is required for cell adhesion and rhombomere-boundary formation in the zebrafish. Curr Biol 15:536-542

Cooper MS, D'Amico LA, Henry CA (1999) Confocal microscopic analysis of morphogenetic movements. Methods Cell Biol 59:179-204

Cunliffe VT (2004) Histone deacetylase 1 is required to repress Notch target gene expression during zebrafish neurogenesis and to maintain the production of motoneurones in response to hedgehog signalling. Development 131:2983-2995

Dornseifer P, Takke C, Campos-Ortega JA (1997) Overexpression of a zebrafish homologue of the Drosophila neurogenic gene Delta perturbs differentiation of primary neurons and somite development. Mech Dev 63:159-171

Echeverri K, Oates AC (2007) Coordination of symmetric cyclic gene expression during somitogenesis by Suppressor of Hairless involves regulation of retinoic acid catabolism. Dev Biol 301:388-403
Gajewski M, Elmasri H, Girschick M, Sieger D, Winkler C (2006) Comparative analysis of her genes during fish somitogenesis suggests a mouse/chick-like mode of oscillation in medaka. Dev Genes Evol 216:315-332

Golling G, Amsterdam A, Sun Z, Antonelli M, Maldonado E, Chen W, Burgess S, Haldi M, Artzt K, Farrington S, Lin SY, Nissen RM, Hopkins N (2002) Insertional mutagenesis in zebrafish rapidly identifies genes essential for early vertebrate development. Nat Genet 31:135-140

Gray M, Moens CB, Amacher SL, Eisen JS, Beattie CE (2001) Zebrafish deadly seven functions in neurogenesis. Dev Biol 237:306-323

Guthrie S, Lumsden A (1991) Formation and regeneration of rhombomere boundaries in the developing chick hindbrain. Development 112:221-229

Haddon C, Smithers L, Schneider-Maunoury S, Coche T, Henrique D, Lewis J (1998) Multiple delta genes and lateral inhibition in zebrafish primary neurogenesis. Development 125:359-370

Hans S, Scheer N, Riedl I, v Weizsacker E, Blader P, Campos-Ortega JA (2004) her3, a zebrafish member of the hairy-E(spl) family, is repressed by Notch signalling. Development 131:2957-2969

Hatakeyama J, Bessho Y, Katoh K, Ookawara S, Fujioka M, Guillemot F, Kageyama R (2004) Hes genes regulate size, shape and histogenesis of the nervous system by control of the timing of neural stem cell differentiation. Development 131:5539-5550

Holley SA, Geisler R, Nüsslein-Volhard C (2000) Control of herl expression during zebrafish somitogenesis by a Delta-dependent oscillator and an independent wave-front activity. Genes Dev 14:1678-1690

Hsiao C-D, You M-S, Guh Y-J, Ma M, Jiang Y-J, Hwang P-P (2007) A positive regulatory loop between foxi3a and foxi3b is essential for specification and differentiation of zebrafish epidermal ionocytes. PLoS ONE 2:e302

Hu C, Diévart A, Lupien M, Calvo E, Tremblay G, Jolicoeur P (2006) Overexpression of activated murine Notch1 and Notch3 in transgenic mice blocks mammary gland development and induces mammary tumors. Am J Pathol 168:973-990

Huppert SS, Ilagan MX, De Strooper B, Kopan R (2005) Analysis of Notch function in presomitic mesoderm suggests a $\gamma$-secretaseindependent role for presenilins in somite differentiation. Dev Cell 8:677-688

Itoh M, Kim CH, Palardy G, Oda T, Jiang Y-J, Maust D, Yeo SY, Lorick K, Wright GJ, Ariza-McNaughton L, Weissman AM, Lewis J, Chandrasekharappa SC, Chitnis AB (2003) Mind Bomb is a ubiquitin ligase that is essential for efficient activation of Notch signaling by Delta. Dev Cell 4:67-82

Jiang Y-J, Brand M, Heisenberg C-P, Beuchle D, Furutani-Seiki M, Kelsh RN, Warga RM, Granato M, Haffter P, Hammerschmidt M, Kane DA, Mullins MC, Odenthal J, van Eeden FJM, Nüsslein-Volhard C (1996) Mutations affecting neurogenesis and brain morphology in the zebrafish, Danio rerio. Development 123:205-216

Jülich D, Lim CH, Round J, Nicolaije C, Schroeder J, Davies A, Geisler R, Consortium TS, Lewis J, Jiang Y-J, Holley SA (2005) beamter/deltaC and the role of Notch ligands in the zebrafish somite segmentation, hindbrain neurogenesis and hypochord differentiation. Dev Biol 286:391-404

Kao HY, Ordentlich P, Koyano-Nakagawa N, Tang Z, Downes M, Kintner CR, Evans RM, Kadesch T (1998) A histone deacetylase corepressor complex regulates the Notch signal transduction pathway. Genes Dev 12:2269-2277

Kimmel CB, Warga RM, Kane DA (1994) Cell cycles and clonal strings during formation of the zebrafish central nervous system. Development 120:265-276

Kimmel CB, Ballard WW, Kimmel SR, Ullmann B, Schilling TF (1995) Stages of embryonic development of the zebrafish. Dev Dyn 203:253-310 
Latimer AJ, Shin J, Appel B (2005) her9 promotes floor plate development in zebrafish. Dev Dyn 232:1098-1104

Le Borgne R, Bardin A, Schweisguth F (2005) The roles of receptor and ligand endocytosis in regulating Notch signaling. Development 132:1751-1762

Leve C, Gajewski M, Rohr KB, Tautz D (2001) Homologues of c-hairyl (her9) and lunatic fringe in zebrafish are expressed in the developing central nervous system, but not in the presomitic mesoderm. Dev Genes Evol 211:493-500

Lorent K, Yeo SY, Oda T, Chandrasekharappa S, Chitnis A, Matthews RP, Pack M (2004) Inhibition of Jagged-mediated Notch signaling disrupts zebrafish biliary development and generates multi-organ defects compatible with an Alagille syndrome phenocopy. Development 131:5753-5766

Ma M, Jiang Y-J (2007) Jagged2a-notch signaling mediates cell fate choice in the zebrafish pronephric duct. PLoS Genet 3:e18

Milan DJ, Giokas AC, Serluca FC, Peterson RT, MacRae CA (2006) Notch $1 \mathrm{~b}$ and neuregulin are required for specification of central cardiac conduction tissue. Development 133:1125-1132

Moens CB, Cordes SP, Giorgianni MW, Barsh GS, Kimmel CB (1998) Equivalence in the genetic control of hindbrain segmentation in fish and mouse. Development 125:381-391

Moens CB, Yan YL, Appel B, Force AG, Kimmel CB (1996) valentino: a zebrafish gene required for normal hindbrain segmentation. Development 122:3981-3990

Nasevicius A, Ekker SC (2000) Effective targeted gene 'knockdown' in zebrafish. Nat Genet 26:216-220

Pasini A, Henrique D, Wilkinson DG (2001) The zebrafish Hairy/ Enhancer-of-split-related gene her6 is segmentally expressed during the early development of hindbrain and somites. Mech Dev 100:317-321

Pasini A, Jiang Y-J, Wilkinson DG (2004) Two zebrafish Notchdependent hairy/enhancer-of-split-related genes, her6 and her4, are required to maintain the coordination of cyclic gene expression in the presomitic mesoderm. Development 131:1529-1541

Qiu X, Xu H, Haddon C, Lewis J, Jiang Y-J (2004) Sequence and embryonic expression of three zebrafish fringe genes, lunatic fringe, radical fringe, and manic fringe. Dev Dyn 231:621-630
Riley BB, Chiang MY, Storch EM, Heck R, Buckles GR, Lekven AC (2004) Rhombomere boundaries are Wnt signaling centers that regulate metameric patterning in the zebrafish hindbrain. Dev Dyn 231:278-291

Schmitz B, Papan C, Campos-Ortega JA (1993) Neurulation in the anterior trunk region of the zebrafish Brachydanio rerio. Roux's Arch Dev Biol 202:250-259

Sieger D, Tautz D, Gajewski M (2003) The role of Suppressor of Hairless in Notch mediated signalling during zebrafish somitogenesis. Mech Dev 120:1083-1094

Takke C, Dornseifer P, von Weizsäcker E, Campos-Ortega JA (1999) her4, a zebrafish homologue of the Drosophila neurogenic gene $E(s p l)$, is a target of NOTCH signalling. Development 126:18111821

Tanigaki K, Nogaki F, Takahashi J, Tashiro K, Kurooka H, Honjo T (2001) Notch1 and Notch3 instructively restrict bFGF-responsive multipotent neural progenitor cells to an astroglial fate. Neuron 29:45-55

Tepass U, Godt D, Winklbauer R (2002) Cell sorting in animal development: signalling and adhesive mechanisms in the formation of tissue boundaries. Curr Opin Genet Dev 12:572-582

Trainor PA, Krumlauf R (2000) Patterning the cranial neural crest: hindbrain segmentation and Hox gene plasticity. Nat Rev Neurosci 1:116-124

Westin J, Lardelli M (1997) Three novel Notch genes in zebrafish: implications for vertebrate Notch gene evolution and function. Dev Genes Evol 207:51-63

Xu Q, Mellitzer G, Robinson V, Wilkinson DG (1999) In vivo cell sorting in complementary segmental domains mediated by Eph receptors and ephrins. Nature 399:267-271

Zecchin E, Conigliaro A, Tiso N, Argenton F, Bortolussi M (2005) Expression analysis of jagged genes in zebrafish embryos. Dev Dyn 233:638-645

Zhang C, Li Q, Jiang Y-J (2007a) Zebrafish Mib and Mib2 are mutual E3 ubiquitin ligases with common and specific Delta substrates. J Mol Biol 366:1115-1128

Zhang C, Li Q, Lim C-H, Qiu X, Jiang Y-J (2007b) The characterization of zebrafish antimorphic mib alleles reveals that Mib and Mind bomb-2 (Mib2) function redundantly. Dev Biol 305:14-27 